\title{
Téoros
}

Revue de recherche en tourisme

\section{Juridictions gouvernementales sur le Saint-Laurent, les lacs et cours d'eau du Québec}

\section{Bernard Michaud}

Volume 6, numéro 2, juillet 1987

Le Saint-Laurent magnétique

URI : https://id.erudit.org/iderudit/1080505ar

DOI : https://doi.org/10.7202/1080505ar

Aller au sommaire du numéro

Éditeur(s)

Université du Québec à Montréal

ISSN

0712-8657 (imprimé)

1923-2705 (numérique)

Découvrir la revue

Citer cet article

Michaud, B. (1987). Juridictions gouvernementales sur le Saint-Laurent, les lacs et cours d'eau du Québec. Téoros, 6(2), 12-14. https://doi.org/10.7202/1080505ar d'utilisation que vous pouvez consulter en ligne. 


\title{
Juridictions gouvernementales sur le Saint-Laurent, les lacs et cours d'eau du Québec
}

\author{
Par Bernard' Michaud*
}

Règle générale, la Couronne est propriétaire du lit des lacs et cours d'eau navigables ainsi que du lit des lacs et cours d'eau non navigables en front des lots riverains concedes depuis le ler juin 1984. Cette règle nous amène à poser la question: quand un cours d'eau est-il navigable? La navigabilité est un critère évalué par les tribunaux seulement et cas par cas. II n'y a done pas de liste de tous les lacs et cours d'eau navigables. On sait toutefois que les tribunaux exigeront que le lac ou cours d'eau navigable ait pu être l'objet d'une navigation commerciale pratique et profitable. Avant d'engager un procès, le citoyen aura d'ailleurs avantage à consulter le ministère québécois de l'Environnement qui émet des opinions administratives sur cette navigabilité. Une autre question se pose: que comprend le cours d"eau, où se termine-t-il sur la berge? On utilise un autre critère pour déterminer la limite du lit d'un cours d'eau; c'est celui de la ligne des hautes eaux. Dans les éaux à maréc, ce critère signifie la limite atteinte par les eaux sur la rive naturelle lors des plus hautes marées du mois de mars; ailleurs, c'est la plus haute élévation des eaux, sans débordement, sur la rive naturelle.

En 1867, l'Acte confédératif a effectué un partage du bénéfice des biens de la Couronne, entre le gouvernement fédéral et le gouvernement provincial. Au premier ont été attribues quelques parcelles alors utilisées à certaines fins publiques précises, de sorte que la quasi-totalité de ces biens a été attribuée au second. Ainsi, au Québec, le lit des lacs et cours d'eau appartenant a la Couronne relève du gouvernement du Québec, sauf pour quelques parcelles telles que les terrains de la Couronne qui étaient utilisés en 1867 par le public à des fins de havre public ( $d^{\prime}$ apress nos recherches, cela se limiterait à une portion de ce que comprennent aujourd'hui les VieuxPorts de Montreal et de Québec). En prin-

\footnotetext{
* Mo Michaud ast epent de recherche at de planification a la Direction du milieu hydrique du ministère de I'Environnemant du Qutbec. En 1976, ii a participe au C.R.D.P. (Université de Montríal) a la redaction de

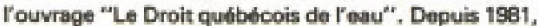
il est charge de cours sur le droit de l'eau A I'T.N.R.S.Eau de Cuabec.
}

cipe donc, le lit des lacs et cours d'eau appartenant à la Couronne a été attribué au gouvernement du Québec. Ces terres constituent ce qu'on appelle le domaine hydrique public et le ministre québécois dé l'Environnement est chargé de les gérer en appliquant la Loi sur le régime des eaux.

\section{Le contexte juridique général}

Le contrôle du domaine

hydrique public

En tant que propriétaire, le gouvernement du Québec détient un important pouvoir de contröle sur le domaine hydrique public. Cependant, la navigation dans les eaux navigables et la pêche (sans fixer d'engin sur le lit) dans les eaux à marée sont deux droits publics qui échappent à ce contrôle. Ces droits publics existaient en 1867 à l'époque de l'Acte confédératif et, depuis, seul le gouvernement féderal est habilité à légiférer à leur sujet. En effet, l'Acte confedératif de 1867, en plus d'effectuer un partage des propriétés de la Couronne, a effectué un partage des juridictions, c'està-dire du droit de légiférer sur une matière donnee. La navigation et les pecheries figurent au nombre des domaines de competence ainsi attribues au gouvernement féderal.

Les domaines de compétence attribués aux deux ordres de gouvernement $n^{\top}$ ont rien à voir avec la propriété des objets réglementés. Par exemple, le fait pour le gouvernement fédéral d'avoir juridiction sur la navigation ne fait pas de celui-ci le proprićtaire des navires, des installations portualres priveses ou encore du lit des cours d'eau navigués. La compétence s'exerce indépendamment de la propriété.

Le droit de péche est une bonne illustration de cette distinction. Sauf pour ce qui est du droit public de péche, c'est-à-dire la pêche dans les eaux à marée sans fixer d'engin sur le lit, le droit de peche est un accessoire du droit de propriété sur le lit. Le propriétaire du lit est le seul à pouvoir pécher et, à son gré, à pouvoir fixer dans ce but des engins sur le lit. Là où le Québec est propriétaire du lit, ee droit lui appartient donc et lui seul peut concéder ce droit. Par contre, toute personne autorisée à pêcher sur le domaine hydrique public devra le faire en respectant les règles édictées par le gouvernement fédéral (saisons, heures, engins, permis, etc.) qui a juridiction exclusive sur les pêcheries. Pour eviter aux citoyens les tracasseries d'une double administration (la Province, pour le droit de pêcher, et l'Etat central, pour le permis de pêche) une entente est intervenue en 1922 entre Ottawa et Québec en matière de peche. La Province administre depuis, pour le gouvernement fédéral, le réglement de ce demier concernant la péche au Quebec. L'attribution du permis fédéral de péche par le Québec accorde en mème temps la permission de pecher dans les eaux où le Québec est propriétaire du droit de péche.

Relativement encore au droit de pêche, signalons que c'est pour conserver ce droit de péche que le gouvernement a instauré la reserve de trois chaines. Avant juin 1884, l'État demeurait proprietaire du lit du cours d'eau lorsqu'il concédait un lot riverain à un lac ou une rivière navigable. Par contre en concédant un lot riverain à un lac ou cours d'eau non navigable, cela impliquait automatiquement la concession du lit jusqu'en son milieu, done la concession du droit de peche sur cette portion. Pour que l'État demeure propriétaire du droit de pecche, une loi est venue faire en sorte que les lots riverains ne soient plus concédés sur une profondeur de trois chaînes ( 3 fois 66 pieds) en bordure des lacs ct rivières non navigables. D'ailleurs, cette réserve a aussi êté êtendue en 1970 aux concessions le long des lacs et rivières navigables. Elle est maintenant de 60 mètres pour les concessions postérieures à décembre 1977. Cette réserve peut cependant ellemème être concédée mais elle doit l'être de façon suffisamment expresse. Cette dernière concession n'implique d'ailleurs plus, depuis une loi de 1918 , la concession automatique du lit d'un lac ou cours d'eau non navigable.

Le fait pour quelqu'un d'être propriétaire riverain lui confere d'importants droits liess à ce statut de propriétaire, tel le droit d'accès, de vue ou d'usage ordinaire de l'eau. Par ailleurs, le fait pour celui-ci 
d'être en plus propriétaire du lit lui confere d'autres avantages, tel le droit de pêche et le droit d'effectuer des travaux et d'ériger des ouvrages sur le lit. Aux endroits où l'État est resté propriétaire du lit, celuici détient ces droits et il faut sa permission pour exercer de telles activités. Dans le cas des travaux et ouvrages, par exemple, cette permission peut être obtenue du ministre de l'Environnement du Québec qui est gestionnaire du domaine hydrique public.

Même lorsqu'un particulier est propriétaire du lit, son droit d'y effectuer des ouvrages peut être subordonné à la nécessité d'obtenir des autorisations. Le droit de propriétés est de moins en moins un droit absolu. Les différentes administrations publiques fédérales, provinciales et municipales édictent, chacune dans son domaine de compétence, un cadre à l'intérieur duquel doivent s'exercer les droits des particuliers.

\section{Contrôle fédéral}

Navigation: parce qu'il a compétence sur la navigation, le gouvernement fédéral doit empêcher que l'érection d'un ouvrage dans les eaux navigables ne puisse nuire à la navigation. Il faut alors une autorisation pour effectuer ces travaux.

Le gouvernement fédéral règle également la circulation des navires dans les eaux navigables et le transbordement des marchandises dans les ports. La navigation de plaisance dans ces eaux doit donc suivre les règles édictées dans le but de ne pas gêner ces activités.

Pêcheries: en vertu de sa juridiction exclusive sur les pêcheries, le gouvernement fédéral interdit les activités qui peuvent avoir un effet néfaste sur l'habitat du poisson (eaux navigables ou non). ${ }^{1}$

Oiseaux migrateurs: en vertu de sa juridiction en matière de relations internationales, le gouvernement fédéral voit avec les provinces à l'application du traité sur les oiseaux migrateurs.

Droit criminel: le comportement des personnes est réglementé par le gouvernement fédéral, par le biais du code criminel. La conduite d'une embarcation avec les facultés affaiblies, la négligence criminelle, la conduite dangereuse, etc., relèvent de cette juridiction.

La défense, les douanes, les eaux interprovinciales ou internationales, les affaires indiennes sont autant de domaines de juridiction qui amènent le gouvernement fédéral à exercer un certain contrôle dans les eaux québécoises.

\section{Contrôle québécois?}

On a parlé précédemment de l'important pouvoir de contrôle du gouvernement qué- bécois en tant que propriétaire du lit de lacs et de cours d'eau.

À ce pouvoir, s'ajoutent ceux que détient le Québec par la juridiction qu'il exerce sur la propriété privée, le droit civil et les matières de nature locale. Ces pouvoirs concernent notamment le droit de circulation sur les eaux et de flottage, le droit d'usage extraordinaire des cours d'eau (pouvoirs hydrauliques, régularisation des eaux, emmagasinement). Ces pouvoirs concernent également les mesures de protection de la qualité des lacs et cours d'eau, et de récupération de cette qualité. Ils concernent de même la gestion des plaines d'inondation, le drainage agricole ou routier, les eaux souterraines, etc.

Enfin, en vertu de la juridiction exclusive qu'il détient sur les municipalités, le Québec exerce un contrôle indirect pas les différents pouvoirs qu'il a attribués aux municipalités. L'hygiène, l'entretien des cours d'eau, le contrôle des embarcations motorisées sont de ce nombre, sans oublier les récents pouvoirs des municipalités locales et régionales de comté (MRC) en matière de zonage, de lotissement et de construction, qui leur permettent notamment de déterminer les grandes orientations et les grandes affectations de leur territoire, de régir ou prohiber la construction ou certains ouvrages à proximité des lacs et cours d'eau.

\section{Application particulière au Saint-Laurent}

Le Saint-Laurent est un cours d'eau navigable. Il est également à marée, en remontant, du golfe jusqu'aux Trois-Rivières. Ces deux caractéristiques nous permettent de particulariser pour le fleuve les règles générales précédemment exposées.

Son lit fait donc partie du domaine hydrique public. Il appartient au gouvernement du Québec et est géré par le ministre de l'Environnement. L'exécution de travaux, l'érection et le maintien d'ouvrages sur ces terrains ne peuvent se faire sans avoir préalablement obtenu du ministre une vente, un bail ou un permis d'occupation.

Exceptionnellement, certaines portions du lit du Saint-Laurent appartiennent à des particuliers ou au gouvernement du Canada. Les premiers ont pu les acquérir suite à une concession expresse de la Couronne. Telle concession a pu se faire par mention à cet effet dans les titres seigneuriaux ou encore par vente sous forme d'acte notarié ou de lettres patentes délivrées par l'administration des terres du domaine public. Quant au gouvernement du Canada, sa propriété sur certaines portions du lit du fleuve lui vient soit d'acquisition, de gré à gré ou par expropriation, de terrains appartenant à des particuliers, soit de transfert par le Québec du contrôle de la régie et de l'administration de terres du domaine hydrique public, soit enfin par l'effet de l'Acte confédératif de 1867.

Bien qu'étant propriétaire du lit du SaintLaurent, le Québec n'a aucun contrôle sur le droit public de navigation dans ses eaux ainsi que sur le droit public de pêche là où la marée se fait sentir. "Le droit public de navigation est essentiellement le droit dont jouit le public de passer et de se déplacer sur les cours d'eau navigables à des fins de transport et de commerce""2. La navigation de plaisance "ne fait pas comme tel partie du droit public de navigation (...) Cependant, la liberté de circulation sur les eaux navigables que crée le droit public de navigation a pour conséquence de la permettre (...) Elle devra manifestement ne pas être exercée de manière à gêner ou obstruer la navigation commerciale"' 3 .

Quant au droit public de pêche, il s'exerce sur le fleuve ainsi que sur ses tributaires, là où la marée se fait sentir, jusqu'à TroisRivières. Ce droit ne comporte pas celui de fixer des engins sur le sol, auquel cas il faudra obtenir l'autorisation du propriétaire du lit. Il faut cette autorisation également pour toute pêche ailleurs dans le fleuve, là où il n'y a pas de marée. Relativement à la pêche sportive, le permis annuel de pêche constitue cette dernière autorisation pour les endroits où le lit appartient au gouvernement québécois et où celui-ci n'y a pas concédé de droits exclusifs (pourvoiries). Pour la pêche commerciale, il faut un permis délivré par le ministre québécois de l'Agriculture, des Pêcheries et de l'Alimentation.

Le droit public de pêche consiste dans le droit de pêcher à la ligne ou à la canne et ligne sans la permission du propriétaire. Par contre, son exercice ainsi que toute activité de pêche doit respecter les règles édictées par le gouvernement fédéral. Celui-ci permet, sans permis, la pêche à la ligne ou à la canne et ligne, en aval du pont Laviolette, pour l'éperlan ou le poulamon.

Pour la chasse aux oiseaux migrateurs, il y a une double juridiction, comme pour la pêche. Elle differe cependant. Le gibier est un "profit à prendre"; il n'appartient pas au propriétaire du terrain. Il faut toutefois la permission de celui-ci pour chasser sur un terrain. De plus, le pouvoir de contrôler la chasse n'a pas été attribué exclusivement au gouvernement du Canada. Le Québec exerce donc le contrôle général de cette activité (permis, quotas, engins, etc.) et donne les autorisations pour chasser sur le domaine hydrique public. Comme plusieurs pays sont concernés par les oiseaux migrateurs, le gouvernement du Canada a signé avec ces pays la Convention concernant les oiseaux migrateurs et lui aussi exerce, en application de la convention, un contrôle sur cette chasse. Les documents distribués annuellement par le gouvernement du Québec sur la réglementation de 
la chasse et de la péche résument bien pour le lecteur les formalités à remplir concernant tout ce qui touche ces deux sujets, pour les deux niveaux de gouvernement.

Le riverain du Saint-Laurent dispose d'un droit d'accès au fleuve où il peut exercer son droit public de navigation incluant celui d'ancrage et d'amarrage, a la condition de ne pas nuire indûment aux autres dans l'exercice de leurs propres droits. En contrepartie, le riverain est soumis à la servitude de halage, c'est-à-dire qu'il doit permettre qu'on utilise une partie de son terrain, en passant, pour des fins de navigation, notamment pour haler les navires.

Le riverain contrôle le passage des tiers qui voudraient accéder au cours d'eau en passant sur sa propriété. Il ne contrồle pas cependant le passage des tiers en front de sa propriété, sur la grève, là où le gouvernement đu Québec est propriétaire, ằ moins de détenir un bail pour cet espace. II lui faut également un bail ou un permis d'occupation selon le cas pour aménager ou ériger des ouvrages, par exemple à des fins de marina, de plage, de site de péche en rive, de quai, d'abri à bateau, etc. De plus, chaque type d'intervention sur le lit du SaintLaurent requiert différentes autorisations. Il y a le permis de construction municipal qui permet de vérifier si l'intervention est conforme à la réglementation municipale et respecte le schéma d'aménagement de la MRC.

Habituellement, une intervention en cours d'eau requiert aussi un certificat d'autorisation délivré par le bureau régional du ministère québécois de l'Environnement. Pour une intervention en zone agricole, l'utilisation d'un terrain a des fins autres que l'agriculture est contrólée par la Commission de protection du territoire agricole. Si l'intervention est susceptible de gêner la navigation, une autorisation devra être obtenue du ministère fédéral des Transports, au service de la Protection des eaux navigables. Si l'intervention a lieu dans les limites administratives d'un port sous juridiction fédérale, il faudra se conformer aux règles édictées par la Société du Port de Montréal (du pont Champlain jusqu'd Repentigny) pour la rive nord, et jusqu'à Tracy pour la rive sud), la Sociéte du Port de Québec (entre St-Nicolas et l'ille d'Orléans), la Société du Port de TroisRivières (les quais de Cap-de-la-Madeleine et de Ste-Angèle-de-Laval et tout le fleuve en front de Trois-Rivières), la Sociêté canadienne des ports pour les ports de Sept-lles (la baie de Sept-Iles) et de Sorel (l'embouchure du Richelieu). Pour les autres ports, il y a la Direction des havres et ports de Transports Canada, pour tous les ports commerciaux ou de traversiers et, enfin, la Direction des ports pour petits bateaux de Pêches et Océans Canada, pour les ports de pốche. Les limites administratives, où ces deux derniers organismes exercent un

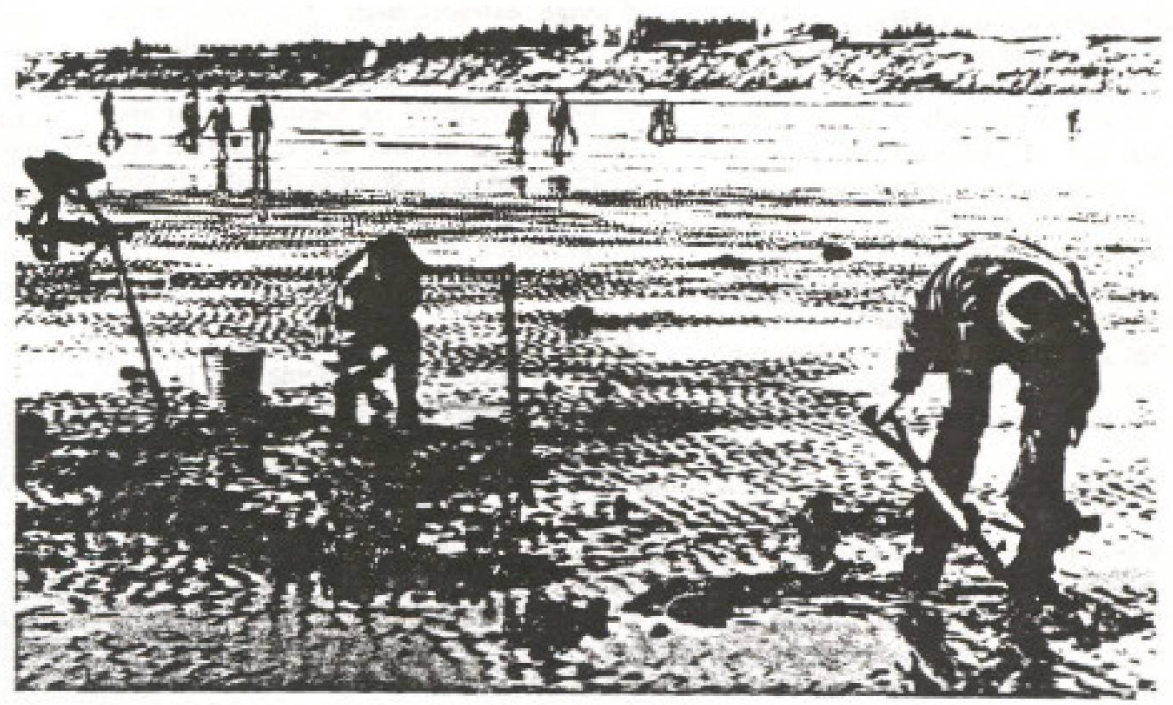

contrôle, ne comprennent habituellement pas plus que l'emplacement des installations portuaires et leurs environs immediats. Par ailleurs, certains secteurs du fleuve, près de Montréal sont sous le controble de la Voie maritime du Saint-Laurent.

\section{Conclusion}

Le citoyen qui entreprend une activite. impliquant une utilisation de l'eau ou devant simplement s'exercer en milieu hydrique, peut avoír plusieurs démarches à engager. $\mathrm{A}$ la fois à cause de leur vulnérabilité et de leur utilité, les lacs et cours d'eau ont été l'objet d'une attention particulière de la part des différents organismes publics concernés. Chacun dans son domaine de juridiction, le gouvernement fédéral et le gouvernement provincial ainsí que beaucoup de leurs ministères et organismes, incluant les instances régionales et locales, sont nombreux à étre concernés par les différentes utilisations des lacs et cours d'eau. Il ne faut donc pas $s^{+} e ́ t o n n e r ~ q u ' u n$ projet, à première vue mineur; puisse interesser Transports-Canada et la Société des ports du Canada (pour la navigation), Péches et Océans Canada (pour le poisson), Environnement-Canada (pour les impacts environnementaux d'un projet sous juridiction fédérale), le ministère québécois de l'Environnement (pour l'utilisation du domaine hydrique public et pour les impacts environnementaux), les ministêres de l'Agriculture, des Pecheries et de 1'Alimentation ainsi que du Loisir, de la Chasse et de la Pêche (pour le poisson), enfin, les municipalités locales et régionales de comté (pour le zonage et la construction).
La localisation du projet, autant que le type de projet lui-même, détermine les autorisations ou permis à obtenir des autorités. publiques. Principales vérifications à effectuer
pour une intervention:

1- Conformité du projet avec la réglementation municipale et le schéma d'aménagement de la MRC.

2- Autorisation du propriétaire du lit (vérifier auprès du ministère québécois de l'Environnement).

3- Autorisations en vertu de la Loi sur la qualité de l'environnement.

4 Autorisations de Transports-Canada pour nuisance à la navigation.

5- En zone agricole, autorisation de la Commission de protection des terres agricoles du Québec.

6- Vérifier sil d'autres organismes que bécois ou fédéraux contrôlent le type d'intervention projeté (ex. Affaires culturelles, dans les environs d'un bien protege).

\section{Rálínences}

(1) Voir â ce sujet le rócent document Politique de gevtion de I'habltat du polswon, du ministore des PAches et Océans (Ottawa)

(2) Le Droit quabseols de Ireas, Centro de recherche en droit publis, ministore des Richesses natureiles, Ouebec (1977), pp. 911-912.

(3) Iden, pp. s12-913. 